\title{
Stochastic collocation-based finite element of structural nonlinear dynamics with application in composite structures
}

\author{
K. Sepahvand ${ }^{\mathrm{a}}$ \\ Department of Mechnaical Engineering, Technical University of Munich, 85748 Garching, Germany
}

\begin{abstract}
Stochastic analysis of structures having nonlinearity by means of sampling methods leads to expensive cost in term of computational time. In contrast, non-sampling methods based on the spectral representation of uncertainty are very efficient with comparable accurate results. In this paper, the application of spectral methods to nonlinear dynamics of structures with random parameters is investigated. The impact of the parameter randomness on structural responses has been considered. To this end, uncertain parameters and the structure responses are represented using the gPC expansions with unknown deterministic coefficients and random orthogonal polynomial basis. The deterministic finite element model of the structure is used as black-box and it is executed on a set of random collocation points. As the sample structure responses are estimated, a nonlinear optimization process is employed to calculate the unknown coefficients. The method has this main advantage that can be used for complicated nonlinear structural dynamic problems for which the deterministic FEM model has been already developed. Furthermore, it is very time efficient in comparison with sampling methods, as MC simulations. The application of the method is applied to the nonlinear transient analysis of composite beam structures including uncertain quadratic random damping. The results show that the proposed method can capture the large range of uncertainty in input parameters as well as in structural dynamic responses while it is too time-efficient.
\end{abstract}

\section{Introduction}

The modeling of real structural systems includes some level of nonlinearity and, as well as, some degrees of uncertainty. The main sources of structural nonlinearity may pre-loaded bilinear springs e.g. bolted flanges with gap, free-play joints, elastomer springs, damping from the polymer materials or due to the aerodynamics of moving objects, large deformation and etc. The predictive capabilities becoming always faster to consider these nonlinearity in simulation but limited by the parameter uncertainties. The uncertainty account for two major sources, i.e. parametric and modeling uncertainties. Former is due to the uncertainty in material and geometry parameters and later due to the lack of information on model, e.g. boundary conditions, mathematical approach. The major challenge is, however, how does these nonlinearity and uncertainty can be quantified and modeled in analytical and numerical simulation.

Even the nonlinear stochastic analysis of such structures with random excitation has been well developed in past decades, predicting nonlinear response under parameter randomness is still a challenging issue [1]. The linear structural behavior including parameter uncertainty has been well developed, cf. [2-5]. On the other side, the most of published works on of stochastic nonlinear dynamics are limited to the application of the sampling-based probabilistic methods, e.g. Monte Carlo (MC), or perturbation methods [6-10]. The meth-

a e-mail: k.sepahvand@tum.de ods are straightforward in application, but limited to the small range of uncertainty and very expensive in the term of computational costs. The non-sampling spectral methods, in contrast, are very efficient and exhibit the same level of accuracy sampling methods.The application of the methods is well known in linear structural dynamics [11-16] where random parameters are approximated by spectral expansions to give a complete characterization of the uncertain parameters.

In this paper, the spectral stochastic finite element method (sSFEM) analysis of the nonlinear structural dynamics with parametric uncertainty is developed. This extension will rely in particular type of structural nonlinearity which can be formulated as separate uncertain term. To this end, the theory of spectral representation of uncertain parameters using generalized polynomial chaos (gPC) expansion is presented. The sSFEM formulation of the structural nonlinear dynamics involving uncertain nonlinear terms is developed. The uncertain nonlinear term is approximated by means of a gPC expansion having deterministic nonlinear functions and random orthogonal basis. Similar expansion is used to represent the FE nodal responses with unknown deterministic coefficients. A collocation based FE analysis, then, provides this facility to use any deterministic numerical simulation procedure to estimate these coefficients. The application of the proposed method is practiced on composite beam with polymer core. The primarily experimental modal analysis of the beam sample shows nonlinear behavior due to increasing the force amplitude. 


\section{Spectral representation of uncertain parameters}

Let $X$ be an uncertain parameter with limited variance $\sigma^{2}<\infty$ in random space $\Omega$, so that

$$
X: \Omega \stackrel{f_{X}(x)}{\longrightarrow} \mathbb{R}
$$

The spectral method allows us to represent the parameter by means of truncated expansion such as the generalized polynomial chaos (gPc)as

$$
X(\boldsymbol{\xi})=a_{0}+\sum_{i=1}^{N} a_{i} \Psi_{i}(\boldsymbol{\xi})=\sum_{i=o}^{n} a_{i} \Psi_{i}(\boldsymbol{\xi})
$$

i which $a_{i}$ are unknown deterministic coefficients, $f_{X}(x)$ is the probability density function $(\mathrm{PDF}), \boldsymbol{\xi}$ denotes vector of random variables $=\left\{\xi_{1}, \xi_{3}, \ldots, \xi_{n}\right\}$, e.g. $\xi_{i} \in$ $N(0,1)$ and $\Psi_{i}(\boldsymbol{\xi})$ are random orthogonal polynomials with $\Psi_{0}=1$. The coefficients are estimated based on minimization of the approximation error on entire random space, known as Galerkin projection, or on sample collocation points in the random space. The details of the both procedure has been discussed in [17]. Multiplying the both side of the Eq. (1) by test function $\Psi_{k}(\boldsymbol{\xi})$ and integrating both side on random space $\Omega$ leads to

$$
a_{i}=\frac{\left\langle X(\boldsymbol{\xi}), \Psi_{k}(\boldsymbol{\xi})\right\rangle}{\left\langle\Psi_{i}(\boldsymbol{\xi})^{2}\right\rangle}, \quad k=0,1, \ldots, n
$$

\section{Nonlinear stochastic finite element analysis}

It is assumed nonlinear term of the structure can be separately represented as $\boldsymbol{G}(\ddot{\boldsymbol{u}}, \boldsymbol{u})$ in the FE model, i.e.

$$
\boldsymbol{M} \ddot{\boldsymbol{u}}+(\boldsymbol{C}+\boldsymbol{H}) \dot{\boldsymbol{u}}+\boldsymbol{K} \boldsymbol{u}+\boldsymbol{G}(\boldsymbol{u}, \dot{\boldsymbol{u}})=\boldsymbol{F}
$$

in which $\boldsymbol{M}$ and $\boldsymbol{K}$ are linear mass and stiffness matrices, $\boldsymbol{C}$ and. $\boldsymbol{H}$ are viscous and structural damping matrices and $\boldsymbol{F}$ is the external force vector. The nodal displacement vector is denoted as $\boldsymbol{u}$. In the case of any uncertainty in the nonlinear term, the stochastic FE model can be given as

$$
\boldsymbol{M} \ddot{\boldsymbol{u}}+(\boldsymbol{C}+\boldsymbol{H}) \dot{\boldsymbol{u}}+\boldsymbol{K} \boldsymbol{u}+\boldsymbol{G}(\boldsymbol{u}, \dot{\boldsymbol{u}}, \boldsymbol{\xi})=\boldsymbol{F}
$$

where random vector $\boldsymbol{\xi}$ is used to represent the structure uncertainty. Accordingly, the nodal displacements are function of the random vector, i.e. $\boldsymbol{u}=\boldsymbol{u}(t, \boldsymbol{\xi})$. The random nonlinear matrix is represented by the spectral expansion as

$$
G(u, \dot{u}, \boldsymbol{\xi})=g_{0}(u, \dot{u})+\sum_{i=1}^{n_{1}} g_{i}(u, \dot{u}) \Psi_{i}(\boldsymbol{\xi})=\boldsymbol{g} \boldsymbol{\Psi}^{T}
$$

The zeroth order term $g_{0}(u, \dot{u})$ denotes the nominal nonlinear term and the coefficient $g_{i}(u, \dot{u})$ are deterministic functions. The same orthogonal basis is employed to represent the propagation of the uncertainty in the structural responses. Hence, the spectral representation of the random nonlinear term and the nodal displacements are written as

$$
\boldsymbol{u}(t, \boldsymbol{\xi})=\boldsymbol{U}_{0}(t)+\sum_{i=1}^{n_{2}} \boldsymbol{U}_{i}(t) \Psi_{i}(\boldsymbol{\xi})=\boldsymbol{U} \boldsymbol{\Psi}^{T}
$$

The expansions in Eqs. (5) and (6) are substituted in stochastic FE model in Eq. (4), this leads to

$$
\boldsymbol{M} \ddot{\boldsymbol{U}} \boldsymbol{\Psi}^{T}+(\boldsymbol{C}+\boldsymbol{H}) \dot{\boldsymbol{U}} \boldsymbol{\Psi}^{T}+\boldsymbol{K} \boldsymbol{U} \boldsymbol{\Psi}^{T}+\boldsymbol{g} \boldsymbol{\Psi}^{T}=\boldsymbol{F}
$$

This discretized equation is the spectral stochastic FE model (sSFEM) of the structure having random nonlinear term and accordingly yields to an additional error due to the random space discretization among the FE discretization error. This error is given by

$$
\begin{aligned}
\varepsilon(t, \boldsymbol{\xi}) & =\boldsymbol{M} \ddot{\boldsymbol{U}} \boldsymbol{\Psi}^{T}+(\boldsymbol{C}+\boldsymbol{H}) \dot{\boldsymbol{U}} \boldsymbol{\Psi}^{T}+\boldsymbol{K} \boldsymbol{U} \boldsymbol{\Psi}^{T} \\
& +\boldsymbol{g} \boldsymbol{\Psi}^{T}-\boldsymbol{F}
\end{aligned}
$$

Generally, there are two broad classes of methods that can be used to minimize the above mentioned error: intrusive and non-intrusive methods. The implementation of intrusive methods requires access to the data structures of the FE model or analytical governing equations, whereas in the non-intrusive method the FE model or the governing equations are employed as third party solver or black-box. The choice between these methods depends on the problem under investigation and the information available on the system model. For instance, if the governing equations of the system are linear and available, the intrusive method can be used to generate the deterministic equivalent model. However, the application of the non-intrusive method to the nonlinear equations is easier.

\subsection{Non-intrusive sSFEM}

The so-called non-intrusive SFEM method looks for a set of deterministic model responses corresponding to some specific points of $\boldsymbol{\xi}$ realizations from which the system response can be approximated. In the concept of stochastic variational, the non-intrusive SFEM method is the same as the collocation method which forces the residual error to be deterministically zero at specific points, i.e. in Eq. (8)

$$
\int_{\left\{\xi_{i}\right\}} \varepsilon(t, \boldsymbol{\xi}) \delta\left(\xi_{i}-p_{i}\right) f\left(\xi_{i}\right) d \xi_{i}=0
$$

In which $\delta$ is the delta function and $p_{i}$ denotes the set of specific collocation points. The method allows the use of third-party commercial or free license deterministic FE codes as a black-box to get the system responses associated with each realization of random vector. These characteristics make the method very attractive for parametric quantification in complex models and industrial applications where the deterministic FE model has been already developed. The key idea is to use deterministic FE codes to perform 
spatial discretization to arrive at a system of random algebraic equations to employ in the SFEM. Normally, the roots of orthogonal polynomials are used as realizations of the random variables. An optimization process, usually based on least squares techniques, is employed if the number of realizations are more than the number of unknown coefficients in the gPC expansion of the system response.

\section{Case study}

In this section the application of the proposed method is implemented to perform the uncertainty analysis in stochastic structural nonlinear vibration of composite beam having random quadratic damping, cf. Fig. 1. The damping core has a thickness of $30 \mu \mathrm{m}$



(a)

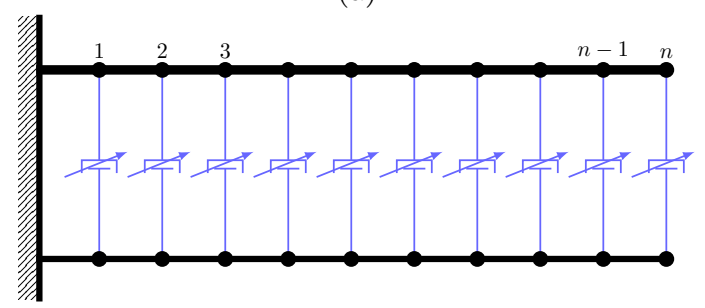

(b)

Figure 1. (a) Double elastic composite beam with polymer core, (b) FE model with nonlinear quadratic damping

and the elastic sheet metals have a thickness of $1 \mathrm{~mm}$. The beam length is set to $180 \mathrm{~mm}$. The experimental modal analysis shows that the composite beam possesses a high damping level by increasing the excitation frequency compared to the simple elastic beam without polymer core. as shown in Fig. 2. The initial experimental modal analysis of the sample beam demonstrates also some level of nonlinearity where the frequency response shows amplitude dependency, see Fig. 3. It is shown that increasing the force amplitude, and accordingly the structural responses, will change the frequency responses and the damping level. The general model of such nonlinear damping is given by

$$
G(\dot{u}, \xi)=c_{s}(\xi) \dot{u}|\dot{u}|^{n-1}
$$

in which $n=0$ denotes the Coulomb friction, $n=1$ represents the classical linear viscous damping and $n=2$ gives nonlinear quadratic damping. It is assumed that the damping coefficient $c_{s}$ has some level of uncertainty which is approximated by the $3^{\text {rd }}$ order gPC expansion having random orthogonal Her-



Figure 2. The composite beam with polymer core shows high damping level compared to the elastic beam without damping layer having the same thickness.



Figure 3. Increasing the force amplitude will change the frequency response and the damping level of the composite beam with polymer core.

mite polynomials, i.e.

$$
c_{s}(\xi)=\sum_{i=0}^{3} a_{i} H_{i}(\xi)
$$

It is also assumed that the uncertainty in the damping coefficient can be represented by the lognormal PDF as $L N(\mu, \sigma)$ with $\mu=1$ and $\sigma=0.25$. The sSFEM uses a deterministic FE model of the double beam as a black-box where the interlayer nodes are connected to each other by discrete nonlinear damping elements. For each realization of the structural response, the collocation points are produced from the combination of roots of one order higher than the order of the gPC expansion used to approximate the responses. The combinations are selected in such a manner to give high probability. The sample points are passed through the deterministic FE code to realize the tip displacement of the beam. Here a set of 17 collocation points is employed. An optimization process based on the least squares is utilized since the number of collocation points is more than the number of unknown gPC coefficients. The deterministic $(\xi=0)$ responses of the beam tip for the linear and 
nonlinear damping are shown in Fig. 4. The difference

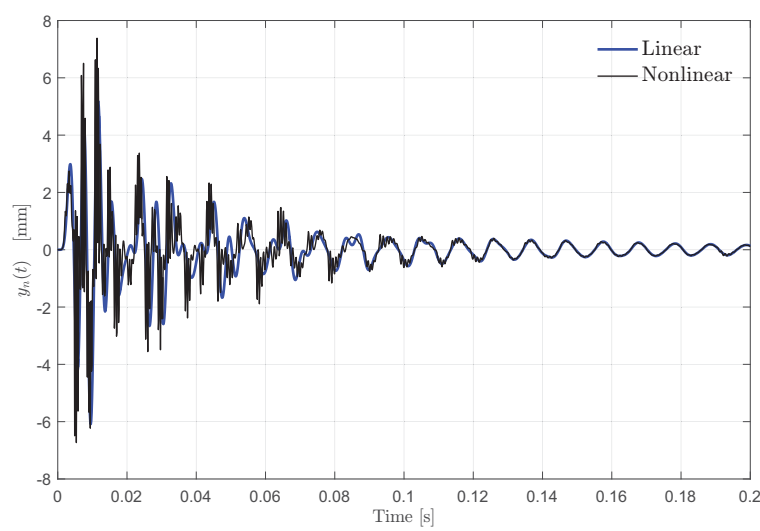

Figure 4. Linear and nonlinear deterministic responses, $\xi=0$.

between the responses in deterministic case is not considerable, particularly, when the time increases. This is however different when damping uncertainties are considered, as shown in Fig. 5. The bold line denotes

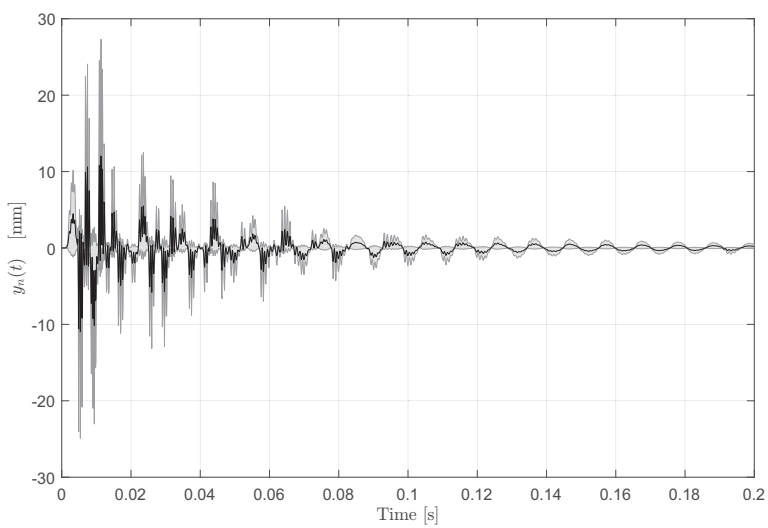

Figure 5. Linear and nonlinear stochastic responses.

the expected value of the response. It is seen that the parameter uncertainty will propagate in stochastic nonlinear responses, shown as gray area in Fig. 5. To investigate the impact of parameter uncertainty, the PDF of the maximum tip displacement is shown in Fig. 6 As shown, the PDF is asymmetry with varying degrees of skewness w.r.t. the mean value shown by the dashed lines. It can be seen that the longer tile ERP is on the right side of the deterministic value, meaning that uncertainties in the damping parameters will increase the level of the displacement.

\section{Conclusions}

The paper presented the theory and application of spectral stochastic FE modeling nonlinear structural dynamics problems with uncertain parameters. Attention was focused on the application of non-sampling probability method in combination with the stochastic

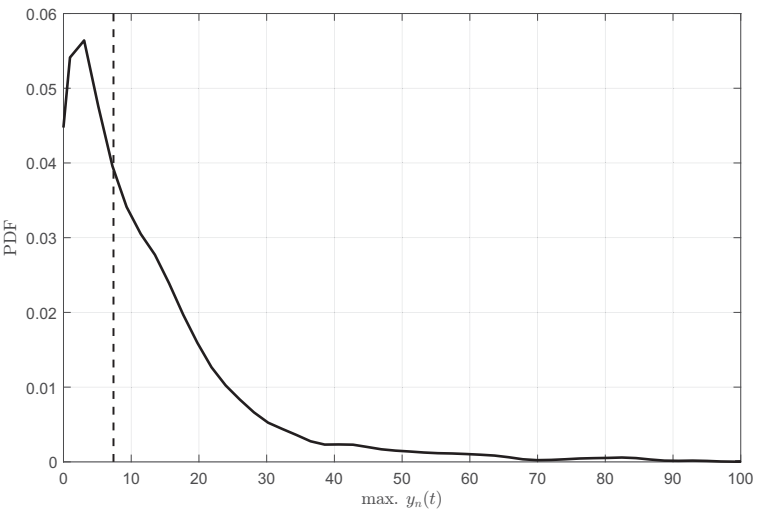

Figure 6. Probability density function (PDF) of the maximum displacement of the beam tip. The dashed line show the position of the deterministic solution.

collocation simulation. The paper aimed to provide a brief review of the uncertainty quantification methods in nonlinear structures using the generalized polynomial chaos expansion. The spectral stochastic FEM formulation for nonlinear structural vibration based on the non-intrusive techniques were developed. The non-intrusive method is useful due to the fact that the available deterministic FE model of the nonlinear structure can be used to investigate the stochastic analysis as black-box. The use of these methods remarkably enhances the computational efficiency. This issue has been pointed out as a significant advantage for numerical simulation of the practical engineering problem. The potential of each technique for numerical simulation of stochastic structural dynamic problems has been discussed on composite beam with polymer core in which the experimental results show some degree of nonlinearity in damping parameters.

\section{References}

1. T. L. Paez and J. Red-Horse. Structural dynamics challenge problem: Summary. Computer Methods in Applied Mechanics and Engineering, 197(2932):2660-2665, 2008.

2. R. A. Ibrahim. Structural dynamics with parameter uncertainties. Applied Mechanics Reviews, 40(3):309-328, 1987.

3. C. Soize. A nonparametric model of random uncertainties for reduced matrix models in structural dynamics. Probabilistic Engineering Mechanics, 15(3):277-294, 2000.

4. M. Hanss. Applied Fuzzy arithmetic, an introduction with engineering applications. SpringerVerlag, Heidelberg, 2005.

5. K. Sepahvand, S. Marburg, and H.-J. Hardtke. Stochastic free vibration of orthotropic plates using generalized polynomial chaos expansion. Journal of Sound and Vibration, 331:167-179, 2012.

6. W. K. Liu, T. Belytschko, and A. Mani. Probabilistic finite elements for nonlinear structural dy- 
namics. Computer Methods in Applied Mechanics and Engineering, 56(1):61-81, 1986.

7. A. Der Kiureghian and J.-B. Ke. The stochastic finite element method in structural reliability. Probabilistic Engineering Mechanics, 3(2):83-91, 1988.

8. Z. Lei and C. Qiu. A stochastic variational formulation for nonlinear dynamic analysis of structure. Computer Methods in Applied Mechanics and Engineering, 190(5-7):597-608, 2000.

9. Y.-G. Zhao and T. Ono. Moment methods for structural reliability. Structural Safety, 23(1):47$75,2001$.

10. J. M. Balthazar, P. B. Goncalves, and R. F. Brasil. Uncertainties in nonlinear structural dynamics. Mathematical Problems in Engineering, 2008:538725-9, 2008.

11. R. G. Ghanem and P. D. Spanos. Stochastic finite elements: A spectral approach. Dover, Mineola, 1991.

12. C. Soize. A comprehensive overview of a nonparametric probabilistic approach of model uncertainties for predictive models in structural dynamics. Journal of Sound and Vibration, 288(3):623652, 2005.

13. K. Sepahvand, S. Marburg, and H.-J. Hardtke. Stochastic structural modal analysis involving uncertain parameters using generalized polynomial chaos expansion. International Journal of Applied Mechanics, 3:587-606, 2011.

14. K. Sepahvand, S. Marburg, and H.-J. Hardtke. Stochastic free vibration of orthotropic plates using generalized polynomial chaos expansion. Journal of Sound and Vibration, 331(1):167-179, 2012.

15. K. Sepahvand, K. Nabih, and S. Marburg. Dynamical analysis of multibody systems with design uncertainties collocation-based stochastic modeling of uncertain geometric mistuning in bladed rotor. Procedia IUTAM, 13:53-62, 2015.

16. K. Sepahvand. Spectral stochastic finite element vibration analysis of fiber-reinforced composites with random fiber orientation. Composite Structures, 145:119-128, 2016.

17. K. Sepahvand, S. Marburg, and H.-J. Hardtke. Uncertainty quantification in stochastic systems using polynomial chaos expansion. International Journal of Applied Mechanics, 2(2):305-353, 2010. 